\title{
The CYP707A Gene Family in Strawberry (Fragaria $x$ ananassa)
}

\author{
Hugo Carlos Bolzon Gonzalez ${ }^{1}$ \\ https://orcid.org/0000-0002-9551-6012 \\ Vanessa Galli ${ }^{1 *}$ \\ https://orcid.org/0000-0002-5735-9749 \\ ${ }^{1}$ Federal University of Pelotas, Technological Development Center, Pelotas, Rio Grande do Sul, Brazil. \\ Editor-in-Chief: Paulo Vitor Farago \\ Associate Editor: Adriel Ferreira da Fonseca
}

Received: 2020.03.05; Accepted: 2021.02.24.

*Correspondence: vane.galli@yahoo.com.br; Tel.: +55-53-32757350 (V.G.)

\section{HIGHLIGHTS}

- Abscisic acid is catabolized by CYP707A enzymes

- CYP707A gene family was characterized in strawberry

- Phylogenetic, network interaction and gene expression analysis were performed

- CYP707A genes are involved in the salt stress response in strawberry.

\begin{abstract}
Abscisic acid ( $A B A)$ is a plant hormone that plays several roles in plant development. The de novo synthesis and the reversible inactivation of $A B A$ have been largely described in the literature; however, the degradation of ABA, promoted by the enzymes Abscisic Acid 8'-Hydroxylase, encoded by the CYP707A gene family, is still poorly elucidated. Strawberry (Fragaria $x$ ananassa) has been used as a model to study the ABA-dependent maturation process of non-climacteric fruits, and the ABA-dependent response to abiotic stress. However, the CYP707A genes from this species have not been fully described and characterized. In this perspective, FaCYP707A sequences were identified from strawberry fruit transcriptome and several structural and comparative genomic analyzes were performed. Moreover, the expression of the FaCYP707A sequences identified was investigated in fruits under salt stress and ABA application. Four putative FaCYP707A were identified and the structural analysis confirmed the identity of three of them. The phylogenetic analysis allowed to determine their homologous in other plant species and to predict their evolutionary history; and the expression profile of the FaCYP707As demonstrated that FaCYP707A3 seems to be involved in the response against salt stress in an ABA-dependent manner. Moreover, the interaction network analysis pointed out proteins involved in the ABA metabolism, heavy metal homeostasis and detoxification, and cell wall dissemble. This study characterized for the first time the CYP707A gene family in F. ananassa; this information will guide future studies in order to develop biofortified fruits and stress tolerant plants.
\end{abstract}

Keywords: gene identification; phylogenetic analysis; stress response; non-climacteric fruits; abscisic acid. 


\section{INTRODUCTION}

The strawberry (Fragaria $X$ ananassa Duch.) is a pseudo fruit of economic importance because its cultivation, processing and commercialization involves several workers. Its high demand is related to the sensorial characteristics, including taste, aroma and color, it is also rich in bioactive compounds, particularly ascorbic acid and phenolic compounds [1-3]. These compounds show health beneficial properties, as antioxidant, antioncogenic, neuroprotective, antimicrobial activity, among others [4].

Despite the high demand of this crop, strawberry is very sensitive to salt stress, with variation on the tolerance rate between different cultivars. Salinity has been pointed as cause for reduction on plant growth and fruit yield, as well as leaf edge burn, necrosis or nutritional imbalance. These effects are influenced by the increasing levels of salinity and by the cultivar tolerance. For example, a recent study [5] compared seven different cultivars of strawberry under salt stress and indicated that "Camino Real", "Benicia", "Chandler", and "Radiance" were less tolerant, while "Albion", "Camarosa", and "San Andreas" were more tolerant.

Previous studies have reported the role of abscisic acid (ABA) in the response of strawberry against salt stress [6-8]. Understand how the content of ABA is regulated in plant cell represents an opportunity to develop more resistant plants. The ABA metabolic pathways can be divided in three: the de novo synthesis pathway is widely described, and is promoted by the NCED enzymes (9-cis epoxycarotenoid dioxigenase); the reversible inactivation by glycosylation pathway is promoted by the enzymes BGs / GTs ( $\beta$-glucosidases / UDP-glucosyltransferases) and have also been described; and the irreversible inactivation pathway is promoted by the enzymes CYP707A (Abscisic Acid 8 '- Hydroxylase 4), and is still poorly characterized. In the irreversible inactivation pathway, ABA is converted to phaseic acid (PA) and later to dehydrophaseic acid (DPA) [9-12].

Regulation of CYP707A has been shown to affect the dynamic balance of $A B A[13,14]$. The increase or loss of function of the CYP707A2 gene in Arabidopsis affects the level of ABA in seedlings, affecting their sensitivity to exogenous glucose [14]. Moreover, the genes PacCYP707A1 and PacCYP707A3 in cherry are positively regulated under water stress and ABA treatment [15]. In Arabidopsis, as in other species, different CYP707A genes plays different roles in plant metabolism, and are regulated according to the tissue, developmental stage, and environmental stimuli [13-16].

For Fragaria ananassa, only one complete sequence of CYP707A gene had been described in the literature by Ji and coauthors [17]. Recently, our research group carried out the assembly and annotation of the transcriptome of strawberry fruits submitted to saline stress and water deficit [6]. Through a deep search in this transcriptome, in the present study we identified four CYP707A genes in Fragaria $x$ ananassa. Our analyses provided detailed information on these FaCYP707As, including phylogenetic tree, genomic structures and network interaction. In addition, the expression profile of FaCYP707A genes was investigated in response to salt and $A B A$ application, providing new information for further studies and biotechnology applications.

\section{MATERIAL AND METHODS}

\section{Identifying possible FaCYP707A sequences in the strawberry transcriptome}

Nineteen CYP707A sequences from three different species (Pyrus pyrifolia, Prunus avium, and Malus $x$ domestica) were searched and selected in GenBank database (https://www.ncbi.nlm.nih.gov/genbank/) and are shown in Table 1. The sequences selected were blasted via Multiblast tool into Fragaria ananassa Camarosa transcriptome obtained previously, aiming to identify possible homologous sequences, using CLC Genomics Workbench 10 - Qiagen® (https://digitalinsights.qiagen.com/products-overview/discoveryinsights-portfolio/analysis-and-visualization/qiagen-clc-genomics-workbench/). 
Table 1. Table showing the list of genes selected from GenBank database to be used in the search for CYP707A homologous in the strawberry transcriptome

\begin{tabular}{|c|c|}
\hline Species & NCBI Identifier \\
\hline Prunus avium & $>$ GU559990.1 \\
\hline Prunus avium & >GU559989.1 \\
\hline Prunus avium & >GU559988.1 \\
\hline Prunus avium & >GU559987.1 \\
\hline Pyrus pyrifolia & >JF825450.1 \\
\hline Pyrus pyrifolia & >LC155802.1 \\
\hline Pyrus pyrifolia & >KP723487.1 \\
\hline Pyrus pyrifolia & >KP723486.1 \\
\hline Pyrus pyrifolia & >KP723485.1 \\
\hline Pyrus pyrifolia & >KP723484.1 \\
\hline Pyrus pyrifolia & >KP723483.1 \\
\hline Pyrus pyrifolia & >KP279631.1 \\
\hline Pyrus pyrifolia & >KP279630.1 \\
\hline Pyrus pyrifolia & $>\mathrm{KP} 162149.1$ \\
\hline Pyrus pyrifolia & >KP162148.1 \\
\hline Pyrus pyrifolia & >KP057206.1 \\
\hline Pyrus pyrifolia & >JN602256.1 \\
\hline MalusX domestica & $>$ AB593331.1 \\
\hline Malus $\times$ domestica & $>$ AB593330.1 \\
\hline
\end{tabular}

\section{Open reading frame (ORF) identification}

The biggest contigs identified in the transcriptome as putative FaCYP707A homologous were submitted to the ORFfinder tool (https://www.ncbi.nlm.nih.gov/orffinder/), using as parameters: Minimal ORF length (nt): $150 \mathrm{nt}$; Genetic code: 1. Standard; ORF start codon to use: 'ATG' only; and Ignore nested ORFs: Not marked. This methodology was applied to obtain the possible open reading frames (ORFs) for each sequence.

\section{Characterization of putative FaCYP707A sequences}

The ORF sequences were used as query in the SmartBlast (https://blast.ncbi.nlm.nih.gov/smartblast/smartBlast.cgi) and BlastP (https://blast.ncbi.nlm.nih.gov/Blast.cgi) tools, aiming to analyze the existence of conserved protein domains, super domains and any other information that may help to identify the protein families, as compared to the sequences already indexed in the NCBI database.

The amino acid sequence for each FaCYP707A was predicted using MEGA $X$ software (https://www.megasoftware.net/). SignalPHMM 5.0 (http://www.cbs.dtu.dk/services/SignalP/), HMMSmart (http://smart.embl-heidelberg.de/) and TargetP 2.0 (http://www.cbs.dtu.dk/services/TargetP/) were then used to search for the presence of any signal peptide and to predict the possible subcellular localization of the proteins.

\section{Alignment of putative FaCYP707A sequences}

The sequences obtained from the ORFfinder were aligned using ClustalW algorithm in the CLC Genomics Workbench 12.0.2.

\section{CYP707A gene sequences search using the Fragaria ananassa Genome}

The same ORF sequences were blasted against the Fragaria ananassa Camarosa Genome, which is assembled as scaffolds and is available at Genome Database for Rosaceae. The CLC Genomics Workbench 12.0.2 software was used to perform this analysis and the sequences found with the highest homology were aligned in the same software, using ClustalW algorithm.

\section{Phylogenetic analysis}

To evaluate the evolutionary history of the CYP707A sequences, the same 19 CYP707A sequences from Pyrus pyrifolia, Prunus avium and Malus $x$ domestica described in Table 1, as well as CYP707A 
sequences from Arabidopsis thaliana (AT4G19230, AT2G29090, AT5G45340 and AT3G19270), and the putative CYP707A from strawberry were aligned using the ClustalW algorithm in the MEGAX software. Then, a phylogenetic tree was built using the neighbor joining method, with bootstrap values of 1000 .

\section{Network Interaction}

STRING 11 (Search Tool for the Retrieval of Interacting Genes/Proteins) is a database that aim to provide analysis of known and predicted protein-protein interactions in an organism, also known as protein network, allowing the study of interactions including direct (physical) as well as indirect (functional) association, on a global scale.

Therefore, a specific interaction network was constructed using textmining, databases, co-expression, neighborhood, gene fusion, co-occurrence and experimental evidence in the online STRING 11 (http://stringdb.org/), using Fragariaxvesca homologous from the putative FaCYP707A genes.

\section{Plant materials and stress treatments}

The study was conducted on a greenhouse, using the cultivar Camarosa. The seedlings with similar crown size (approximately $9 \mathrm{~mm}$ diameter) were planted and grown in $9 \mathrm{~L}$ pots, containing soil (Ultisoil) and vermiculite (in a proportion of 3:1). The fertilization and irrigation were performed according to Galli and coauthors. [18]. The relative humidity in each pot was monitored using a hygrometer in order to maintain between $16 \%$ and $19 \%$, without water leaching. The experiment was composed by four treatments with six replicates per treatment and ten plants per replicate. The plants were subjected to the following treatments: C - Control; SS - Salt Stress; ABA+SS - exogenous application of $200 \mu \mathrm{M}$ ABA + Salt Stress (SS); ABA exogenous application of $200 \mu \mathrm{M}$ ABA. For SS, $50 \mathrm{~mL}$ of $400 \mathrm{mM} \mathrm{NaCl}$ was applied once a week. The stress level was based on preliminary experiments. ABA was obtained by Sigma Aldrich and was applied weekly by foliar spraying. The ABA and SS treatments were performed from the beginning of the flowering stage (45 days after transplanting) to the end of the experiment (95 DAT). An approximate pool of ten ripe fruits (fully red, according to Jia and coauthors. [19]) by replicate were harvested. All samples were immediately frozen in liquid nitrogen and stored at $-80^{\circ} \mathrm{C}$ until further analysis.

\section{Gene expression analysis}

To analyze the effects of salt stress and/or ABA exogenous application on CYP707As gene expression, the total RNA from strawberry mature fruits was isolated using CTAB (cetyltrimethylammonium bromide) according to Galli and coauthors. [18]. Meanwhile, aiming to evaluate the pattern of expression in the different development stages, samples were collected in six periods, representing: 7 (small green, SG), 14 (big green, BG), 18 (degreening, DG), 21 (white, W), 24 (partial red, PR), and 28 (full red, FR) days after anthesis (DAA), as previously reported [19]. Fifteen uniformly sized fruits at each stage were collected and separated into three pools (5 fruits for each repetition). Samples of Leaf $(L)$ and Root $(R)$ from five different plants were also collected, immediately frozen in liquid nitrogen and stored at $-80^{\circ} \mathrm{C}$ until analysis. The RNA concentration was quantified using NanoVue ${ }^{\circledR}$. Total RNA was treated with DNAse and reverse transcribed using the MMLV enzyme and oligo-dT primers, according to the manufacturer's instructions (Invitrogen). cDNA was obtained according to Galli and coauthors. [18]. The reference genes PIRUV_DESCARB (pyruvate decarboxylase), DBP (DNA binding protein), and HISTH4 (histone H4) were used to normalize transcription levels, as proposed by Galli and coauthors. [18]. The primers used are presented the same as Perin and coauthors. [7]. The relative expression data were calculated according to the $2^{-\Delta \Delta C q}$ method. The analysis was performed for four biological replicates and three analytical replicates. The results were submitted to analysis of variance (ANOVA) and when significant $(p \leq 0.05)$ were submitted to a mean comparison using Tukey test at $5 \%$ of probability. Statistical analyzes were performed using SAS software.

\section{RESULTS}

\section{Identifying possible FaCYP707A sequences in the strawberry transcriptome}

The search for FaCYP707A genes using homologous sequences from other species allowed the identification of four putative coding sequences for FaCYP707A in strawberry: CL15525Contig1 (FaCYP707A1), CL17770Conting1 (FaCYP707A2), CL18454Contig1 (FaCYP707A3) and CL24483Contig1

(FaCYP707A4). The CL15525Contig1 (FaCYP707A1) sequence was previously described by Jia and coauthors. [19]. 


\section{ORF identification}

In the ORFfinder tool, only the biggest ORF sequence for each contig found in the transcriptome were selected. The FaCYP707A1 had an ORF sequence of $1446 \mathrm{nt}$, and was the longest sequence identified. The shortest sequence was from FaCYP707A4 which had an ORF sequence of 291 nt. Each FaCYP707A and their respective longest ORF sequence were described in Table 2.

Table 2. The ORF information for each FaCYP707A sequence.

\begin{tabular}{ccccccc}
\hline CONTIG & TOTAL NT $^{1}$ & FRAME & Initial & End & ORF NT $^{2}$ & ORF AA $^{\mathbf{3}}$ \\
\hline FaCYP707A1 & 1753 & -3 & 1670 & 225 & 1446 & 481 \\
FaCYP707A2 & 1603 & -3 & 1520 & 225 & 1296 & 431 \\
FaCYP707A3 & 895 & -2 & 840 & $>1$ & 840 & 279 \\
FaCYP707A4 & 653 & -2 & 484 & 194 & 291 & 96 \\
\hline
\end{tabular}

1 - Total Nucleotides; 2 - ORF Nucleotides; 3 - ORF amino acids.

\section{Characterization of putative FaCYP707A sequences}

By using the SmartBlast, it was possible to identify the sequences with higher identity and coverage among the sequences available in the database. FaCYP707A3 showed higher similarity with a sequence from Arabidopsis thaliana, while FaCYP707A1 and FaCYP707A2 showed higher similarity with a Glicine max sequence.

The length of FaCYP707A1 and FaCYP707A2 sequences, compared to similar sequences in the Genbank database, suggests that they represent complete sequences. Moreover, for all sequences the leading match was with Abscisic Acid 8' - Hydroxylase, assuring their identification as CYP707A sequences. The matching sequences shown by SmartBlast are described in Table 3.

Table 3. Data shown by the SmartBlast search for each FaCYP707A sequence.

\begin{tabular}{cccccc}
\hline Contig & Species match & Description & Cover & Identity & E-value \\
\hline FaCYP707A1 & Glycine max & abscisic acid 8'-hydroxylase 4-like & $99 \%$ & $71 \%$ & 0.0 \\
FaCYP707A2 & Glycine max & abscisic acid 8'-hydroxylase 4 & $99 \%$ & $64 \%$ & 0.0 \\
FaCYP707A3 & $\begin{array}{c}\text { Arabidopsis } \\
\text { thaliana }\end{array}$ & $\begin{array}{c}\text { cytochrome P450, family 707, } \\
\text { subfamily A, polypeptide 4 }\end{array}$ & $100 \%$ & $73 \%$ & $1 \mathrm{e}-154$ \\
FaCYP707A4 & Glycine max & abscisic acid 8'-hydroxylase 4 & $97 \%$ & $80 \%$ & $1 \mathrm{e}-50$ \\
\hline
\end{tabular}

The BlastP and HMMSmart search was able to identify the presence of a P450 super domain and a CypX domain in the sequences of FaCYP707A1, FaCYP707A2 and FaCYP707A3, showing a gap in the middle of the $\mathrm{P} 450$ super domain from FaCYP707A2 sequence.

The SignalPHMM 5.0 and TargetP 2.0 query showed the presence of a signal peptide in FaCYP707A1 and FaCYP707A2 among with a transmembrane region between the amino acid 2 and 21 of the sequences.

\section{Alignment of putative FaCYP707A sequences}

FaCYP707A2 has a gap of 150 nucleotides compared to FaCYP707A1. This gap is located between nucleotide 556 and 705 of the major sequence. On the other hand, FaCYP707A3 and FaCYP707A4 sequences show a region of homology with FaCYP707A1 and FaCYP707A2, from nucleotide 743 to 1415; in the rest of these sequences, only a few dispersed homology nucleotides were observed throughout the sequences.

\section{FaCYP707A gene sequence search using the Fragaria ananassa Genome}

The search for FaCYP707A sequences in the Fragaria ananassa cultivar Camarosa Genome showed that FaCYP707A1 and FaCYP707A2 matched with almost the same sequences, while FaCYP707A3 
matched with completely different sequences. FaCYP707A4 matched with some of the same sequences as FaCYP707A1 and FaCYP707A2 and with other sequences not matched by the other FaCYP707A. The matches for each CYP0707A in the Fragaria ananassa Genome are shown in Table 4.

Table 4. Each FaCYP707A sequence and their respective matches in the Fragaria ananassa Genome.

\begin{tabular}{ll}
\hline CYP707A & IDENTIFIER \\
\hline & >ORD_ID5978 \\
CYP707A1 & >ORD_ID65946 \\
& >ORD_ID87415 \\
& >ORD_ID86795 \\
\hline & OORD_ID5978 \\
CYP707A2 & ORD_ID65946 \\
& >ORD_ID87415 \\
& >ORD_ID86795 \\
\hline \multirow{2}{*}{ CYP707A3 } & >RD_ID90279 \\
& >ORD_ID67618 \\
& >ORD_ID3487 \\
& >ORD_ID5978 \\
& >ORD_ID87415 \\
CYP707A4 & >ORD_ID86795 \\
& >ORD_ID65946 \\
& >ORD_ID5966
\end{tabular}

\section{Phylogenetic analysis}

As shown in Figure 1, FaCYP707A1 and FaCYP707A2 are in the same clade as PaCYP707A4 (>GU559989.1) from Prunus avium and PpCYP707A2 from Pyrus pyrifolia of 2 different cultivars (Mixue and Huanghua - >KP723484.1 and >KP162148.1 respectively). Otherwise, FaCYP707A3 is in the same clade as MdCYP707A1 (>AB593330.1) and PpCYP707A1 from Pyrus pyrifolia of the 2 different cultivars (Mixue and Huanghua - >KP723483.1 and >KP057206.1, respectively). These results suggest that FaCYP707A3 diverged in the evolution history before FaCYP707A1 and FaCYP707A2. The placing of FaCYP707A4 next to the Arabidopsis thaliana sequence (>NC_003071.7) seems to represent a mistake in the align algorithm because the FaCYP707A4 is the smallest sequence used in the analysis, and the $A$. thaliana sequence is the biggest, suggesting that in the lack of a true match with other CYP707A sequence, the algorithm understood that those sequences are close related. 


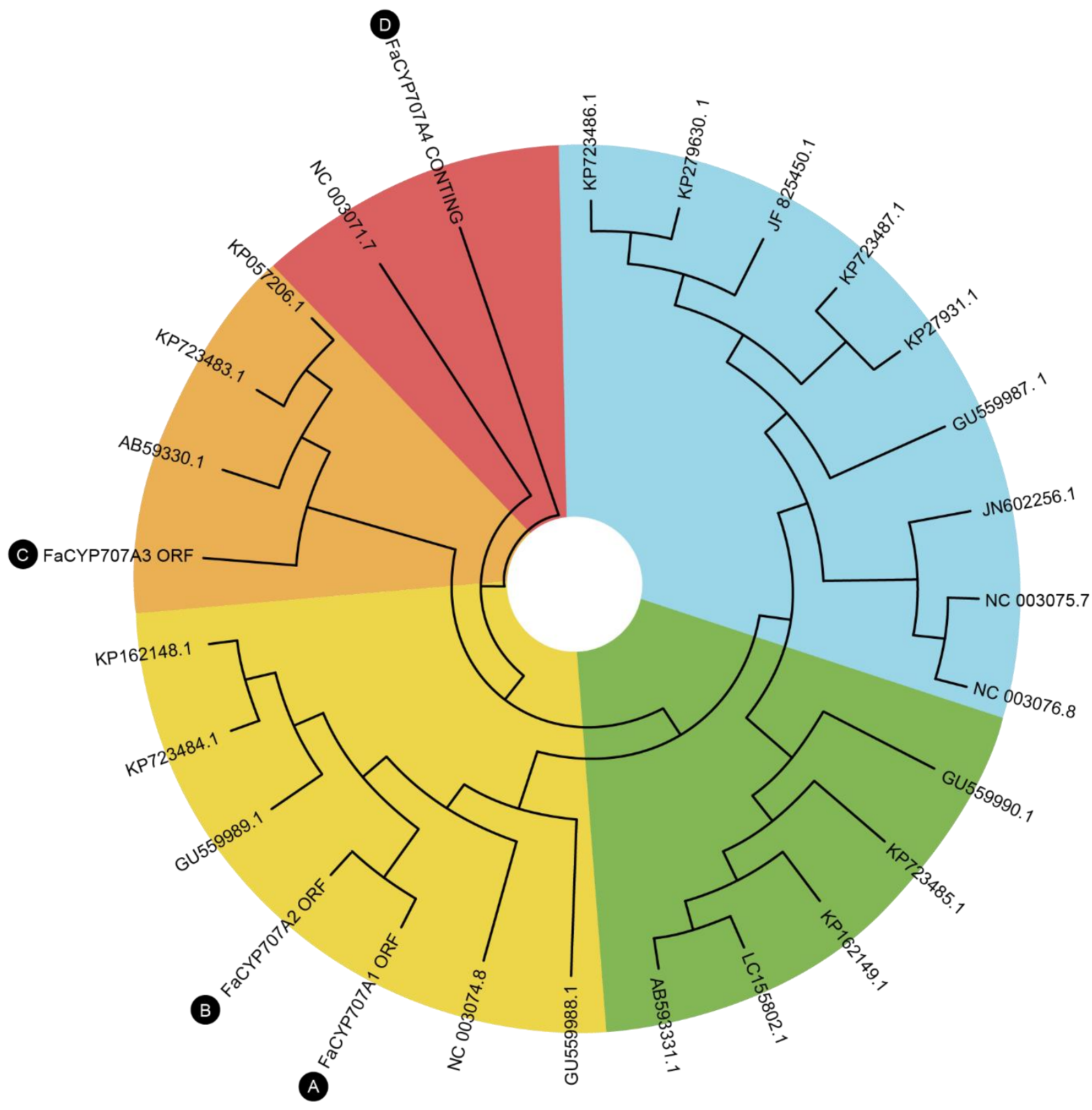

Figure 1. Phylogenetic tree of FaCYP707A genes built with ClustalW algorithm and Neighbor Joining method.

\section{Network Interaction}

When the amino acid sequences of FaCYP707A1, FaCYP707A2, FaCYP707A3 and FaCYP707A4 from Fragaria ananassa were submitted to STRING 11.0 Database, the Fragaria vesca sequences with greater homology for each sequence were presented. FaCYP707A1 and FaCYP707A2 showed greater identity with the sequence Abscisic Acid 8'-Hydroxylase 3 (XP_004291107.1), being 97\% for FaCYP707A1 and 87\% for FaCYP707A2. FaCYP707A3 showed greater homology (99\%) with the Abscisic Acid 8'-Hydroxylase 4 sequence (XP_004294805.1). FaCYP707A4 showed no homology with any sequence present in the database. In addition, FaCYP707A1, FaCYP707A2 and FaCYP707AA3 showed partial homology with two other sequences: Abscsic Acid 8'-Hydroxylase 1 (XP_004300683.1) and Abscisic Acid 8'-Hydroxylase 2 (XP_004295971.1). The interactome obtained in this study is presented in Figure 2. 


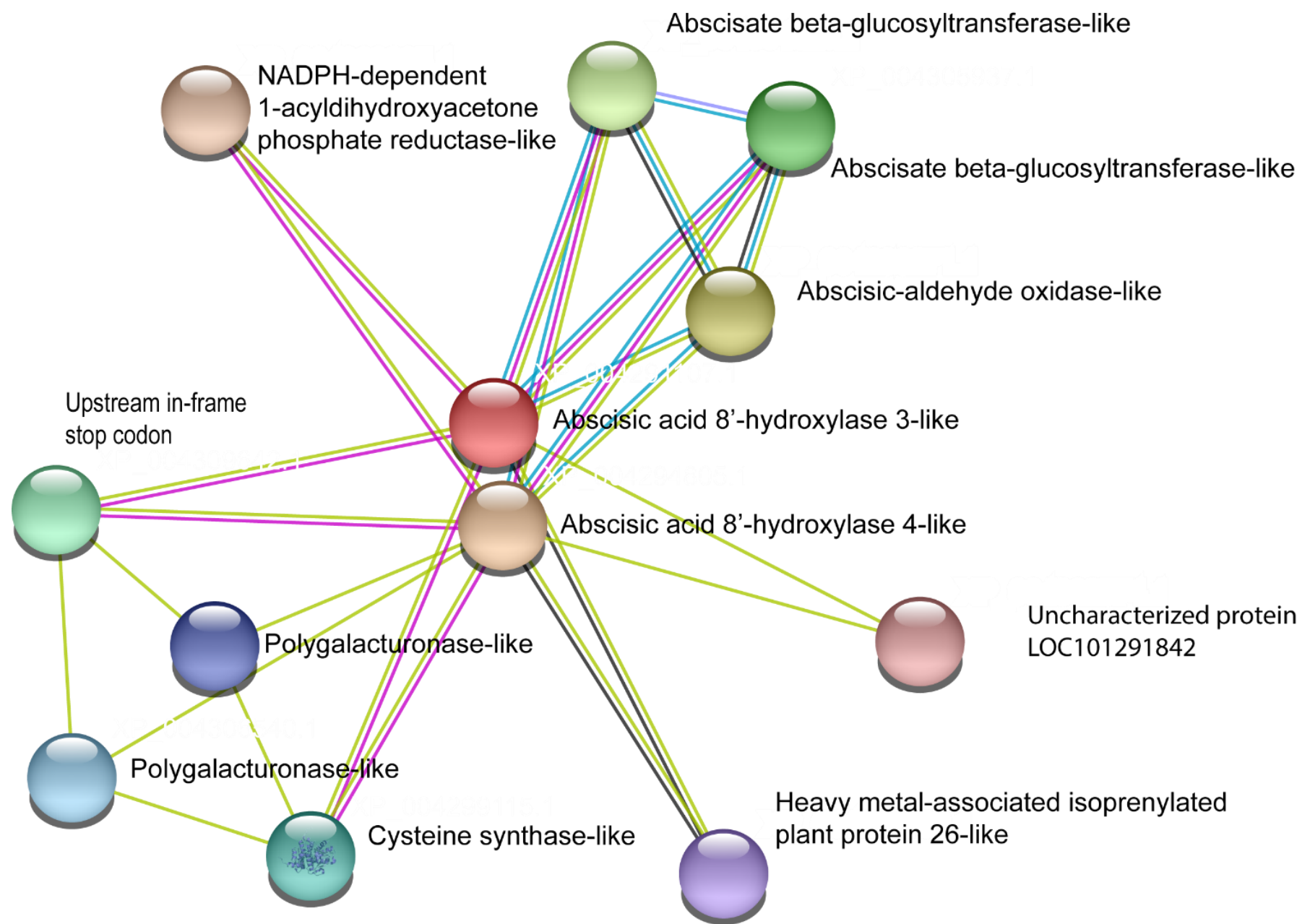

Figure 2. Network interactions of Fragaria vesca sequences homologous to FaCYP707A1-A2-A3, obtained by STRING 11.0 DB tool.

Observing the constructed interactome, the homologous sequence for FaCYP707A1, FaCYP707A2 and FaCYP707A3 in the Fragaria vesca database showed the same correlations with the other proteins present in the construct. Among those, it is interesting to note the textmining and co-expression relation with an HIPP - Heavy metal-associated isoprenylated plant protein 26-like; the textmining correlations with Abscisate betaglucosyltransferase-like proteins, and Abscisic-aldehyde oxidase-like protein, whose putative homologs are mentioned for other species and the interaction is known in curated databases, assuring the association of the CYP707A sequences with the ABA metabolic pathway.

The interactome also shows textmining associations with Cysteine synthase-like protein, Polygalacturonase-like protein and NADPH-dependent 1-acyldihydroxyacetone phosphate reductase-like protein.

\section{Gene expression analysis}

It was possible to observe that the application of salt stress resulted in increased expression of the gene FaCYP707A3 but had the opposite effect on FaCYP707A1 and FaCYP707A2. When ABA was applied to plants subjected to stress, there was a reduction in the expression of FaCYP707A1 and FaCYP707A2, while FaCYP707A3 showed a similar expression profile as the stressed plants not subjected to the application of this phytohormone. Plants supplemented with ABA without stress application showed an increase in FaCYP707A3 expression (Figure 3). The FaCYP707A4 gene showed expression under the limits of RT-PCR detection in strawberry fruits, suggesting that its role may be related to other tissues and physiological processes, and is not represented in the graphs. 
CYP707A1

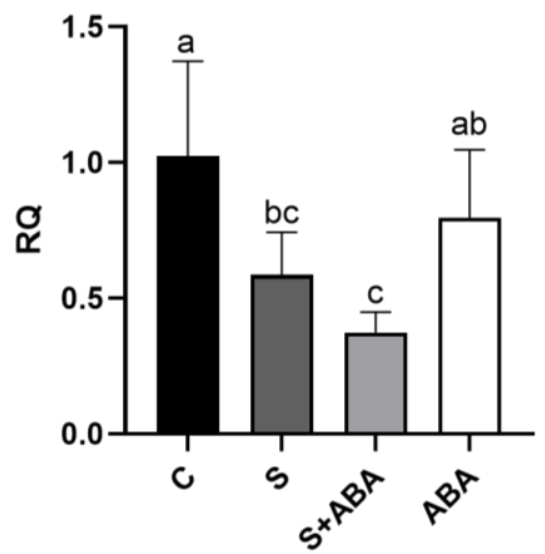

CYP707A2

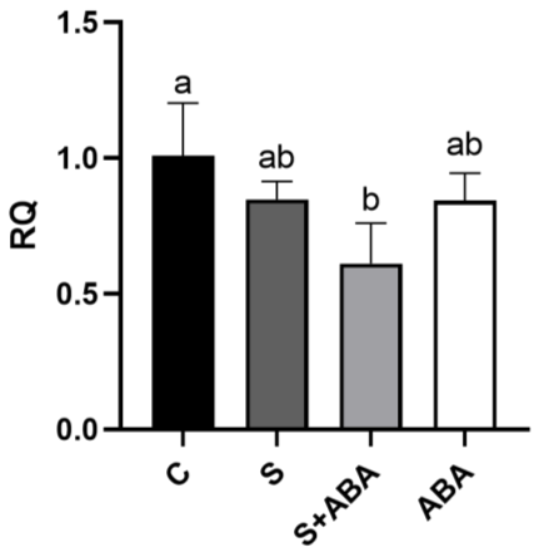

CYP707A3

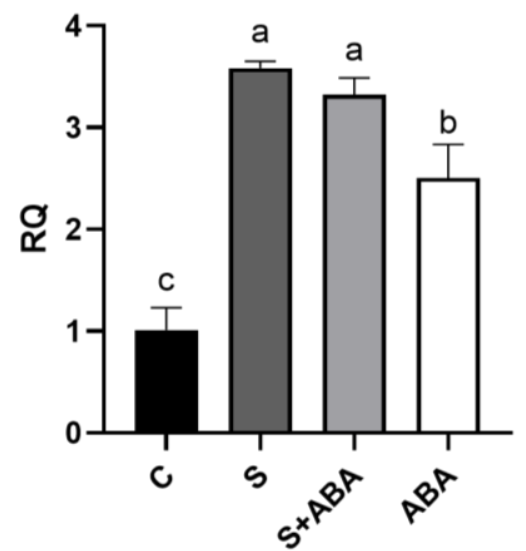

Figure 3. Relative expression of FaCYP707A1, FaCYP707A2 and FaCYP707A3 genes under salt stress and/or the application of ABA.

The expression of FaCYP707A1 during strawberry fruit development was previously described [17]. In the present study we evaluated the expression of the other three FaCYP707A genes to compare with those reported for FaCYP707A1. FaCYP707A2 and FaCYP707A3 showed a significant expression level on day 7, which decreased along fruit development and ripening. The highest expression levels of FaCYP707A2 was observed in fruits at day 7, while the expression of FaCYP707A3 was higher in root samples, and FaCYP707A4 showed the highest expression values in leaves, followed by roots. These results indicate a possible tissue-specific role of different FaCYP707A and during the strawberry fruit development.

CYP707A2

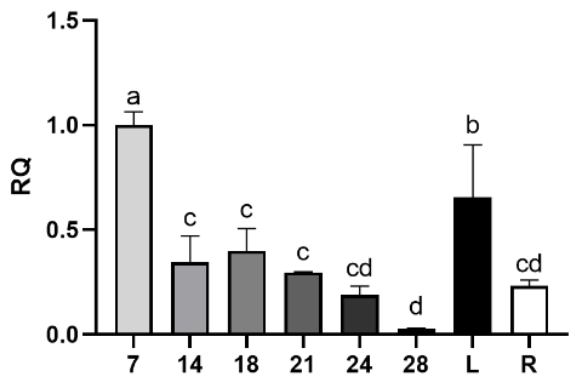

CYP707A3

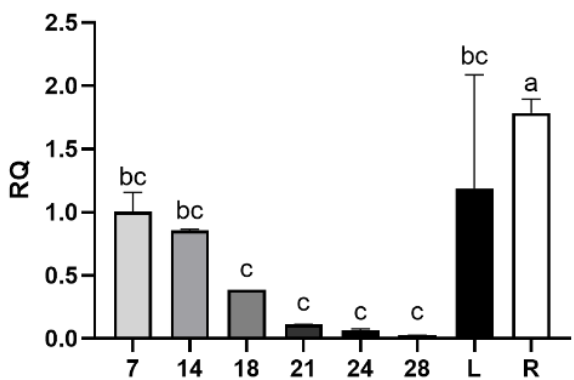

CYP707A4

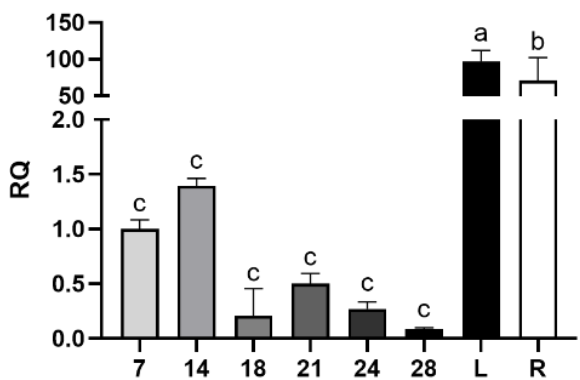

Figure 4. Relative expression of FaCYP707A2, FaCYP707A3 and FaCYP707A4 genes during different stages of fruit development and ripening $(7,14,18,21,24$ and 28 days after anthesis), and in two different tissues ( $\mathrm{L}$ - Leaf and $\mathrm{R}$ Root).

\section{DISCUSSION}

It is well-known that abscisic acid (ABA) plays important role in regulating the maturation of nonclimacteric fruits and in the response against stress in several plant species [10, 19-20]. The enzymes and the role of the de novo synthesis of $A B A$ in different physiological processes have been largely described in the literature; however, the catabolic pathway, where ABA is converted to PA and DPA by CYP707As enzymes is poorly elucidated. Through several analyzes of structural and comparative genomics, the present study describes the FaCYP707As from strawberry, determines their evolutionary relationships and predicts their functions to guide future studies of functional genomics.

According to the sequence alignment, it is possible to observe that FaCYP707A2 is homologous to FaCYP707A1, but with a deletion of 150 nucleotides close to middle of the sequence. This result could represents an error during sequencing or assembly; however, it is also possible that these sequences are a result of alternative splicing that emerged during the process of genes differentiation, since both showed $100 \%$ homology to only one genomic sequence (>ORD_ID5978). More precise studies on these sequences are needed to validate these hypothesis.

The FaCYP707A3 has correspondence with three sequences in the database that did not matched with FaCYP707A1 / A2 / A4, indicating that probably it is a true FaCYP707A sequence, with different evolutionary history and possibly performs specialized functions in this plant. When the amino acid sequence was 
compared with known sequences from Fragaria vesca, the FaCYP707A3 sequence shows the highest homology with FVCYP707A4 (XP_004294805.1) with an identity of 99\%, while FaCYP707A1-A2 show higher homology with $F V C Y P 707 A 3$ (XP_004291107.1) with an identity of $97 \%$ and $87 \%$, respectively.

FaCYP707A4 is the shortest sequence of the four CYP707As identified, and do not presents a P450 domain. Additional studies are necessary using other transcriptomes and databases to evaluate whether this is an additional gene copy that was subjected to gene fragments deletions and possibly changed function during the course of the evolutionary process or it represents the result of alternative splicing, such as FaCYP707A1-A2 sequences.

The sequence alignment with CYP707As from Fragaria vesca has shown the existence of five other sequences of partial homology to the sequences identified in Fragaria ananassa, suggesting the existence of other CYP707A copies in Fragaria ananassa that were not identified via mRNA-Seq database. Therefore, further studies with other mRNA-Seq databases, obtained from different plant tissues and under different experimental conditions are needed to elucidate the existence of other CYP707A sequences in cultivated strawberry and the role of these sequences in different tissues and physiological processes.

The assembly of phylogenetic trees is a valuable tool to understand the origin and evolutionary drift of genetic sequences, providing information regarding the function of each sequence by comparing to sequences from other species whose function are better characterized. Ren and coauthors. [15] demonstrated that during the development of the Prunus avium fruit, the different CYP707A sequences show variation in the rate of gene expression, fulfilling overlapping roles over time. The exogenous application of ABA increased the expression of PaCYP707A1 and PaCYP707A3, while the expression of PaCYP707A4 (sequence > GU559989.1-with greater similarity with FaCYP707A1 and FaCYP707A2) decreased. Kondo and coauthors. [21] demonstrated that MdCYP707A1 (> AB593330.1-with greater similarity to FaCYP707A3) shows upregulation when subjected to water stress and dehydration in apple fruit. Moreover, when exposed to water stress, the PaCYP707A1 and PaCYP707A3 copies showed a significant increase in their expression rates, while no differences were observed in PaCYP707A2 and PaCYP707A4 in dehydrated and control fruits. The sequences also showed variations in gene expression rates associated with the analyzed tissue and the plant's development phase, as well as presenting a different response to water stress and exogenous ABA application. Similar behavior was observed in the present study, where the expression rate in response to water stress and exogenous ABA application ranged between gene copies. FaCYP707A1 and FaCYP707A2 are slightly downregulated when applying stress or exogenous ABA application, and are even more downregulated when subjected to stress and exogenous ABA application simultaneously. Meanwhile, FaCYP707A3 shows upregulation under salt stress and/or exogenous ABA application indicating that the understanding of homologous enzymes in other species may provide an indicative of the role of the sequences being studied. Moreover, while previous study reported that FaCYP707A1 showed increased level from 18 to 21 DAA, decreasing as it ripened [17], the present study showed that the expression of FaCYP707A2, FaCYP707A3 and FaCYP707A4 are reduced along fruit development, suggesting a role during this process; and that FaCYP707A3 are more expressed in root and FaCYP707A4 in roots and leaves, compared to fruits, corroborating the idea of particular/specialized responses and tissue-specific role of the sequences.

Interactome analyzes show textmining interactions between CYP707As and HIPP proteins, which are known for their involvement in homeostasis and detoxification of heavy metals in plants, especially cadmium, in addition to acting in response to thermal and water stress, suggesting that their relationship with CYP707As is related to the response to environmental changes. Also, interaction with Polygalacturonase is present, an enzyme that acts in the maturation process by degrading pectin, promoting structural changes and softening of the fruits. The relationship between polygalacturonases and the ABA pathway has already been demonstrated, where the exogenous application of ABA or Abz (abscinazole - CYP707A inhibitor) results in greater activity of polygalacturonases and reduced firmness of the treated fruits [22].

\section{CONCLUSION}

This study allowed the identification for the first time of FaCYP707A in $F$. ananassa. Four sequences were identified in RNAseq databanks and the genomic structure was characterized, confirming the identity of three of them. A phylogenetic analysis allowed to determine their homologous with other plant species and predicts their evolutionary history. The expression profile of the FaCYP707As demonstrated their specific role according to the physiological condition: FaCYP707A3 seems to be involved in the response to salt stress in an ABA-dependent manner; and FaCYP707A2, FaCYP707A3 and FaCYP707A4 play a role during fruit development, as their expression are reduced along fruit development. Moreover, the interaction network 
analysis pointed out proteins involved in the ABA metabolism, heavy metal homeostasis and detoxification, and cell wall dissemble. Future functional genomic studies will allow elucidating the role of FaCYP707Aa in $F$. ananassa in order to develop biofortified fruits and stress tolerant plants.

Funding: This research was funded by Coordenação de Aperfeiçoamento de Pessoal de Nível Superior - Brasil (CAPES) - Finance Code 001, and Conselho Nacional de Desenvolvimento Científico e Tecnológico, grant number 454970/2014-5, 301187/2018-5 and 422359/2018-1.

Conflicts of Interest: The authors declare no conflict of interest. The funders had no role in the design of the study; in the collection, analyses, or interpretation of data; in the writing of the manuscript, or in the decision to publish the results.

\section{REFERENCES}

1. Schaart JG, Dubos C., De La Fuente IR, et al. Identification and characterization of MYB-b HLH-WD 40 regulatory complexes controlling proanthocyanidin biosynthesis in strawberry ( $F$ ragariax ananassa) fruits. New Phytologist. 2013 Jan;197(2):454-67.

2. Giampieri F, Tulipani S, Alvarez-Suarez JM, Quiles JL, Mezzetti B, Battino M. The strawberry: composition, nutritional quality, and impact on human health. Nutrition. 2012 Jan;28(1):9-19.

3. Li, D, Zhang X, Li L, et al. Elevated $\mathrm{CO} 2$ delayed the chlorophyll degradation and anthocyanin accumulation in postharvest strawberry fruit. Food Chem. $2019 \mathrm{Jul} ; 285(1): 163-70$.

4. Afrin S, Gasperrini M, Forbes-Hernandez T, et al. Promising Health Benefits of the Strawberry: A Focus on Clinical Studies. J. Agric. Food Chem. 2016 Jun;64(22):4435-49.

5. Sun Y, Niu G, Wallace R, Masabni J, Gu M. Relative salt tolerance of seven strawberry cultivars. Horticulturae. 2015 Dec;1(1):27-43.

6. Galli V, Messias R, Guzman F, Perin EC, Margis R, Rombaldi CV. Transcriptome analysis of strawberry (Fragariax ananassa) fruits under osmotic stresses and identification of genes related to ascorbic acid pathway. Physiol. Plant. 2019 Oct;166(4):979-95.

7. Perin EC, Messias RS, Borowski JM, et al. ABA-dependent salt and drought stress improve strawberry fruit quality. Food Chem. 2019 Jan;271(15):516-26.

8. Li D, Mou W, Luo Z, et al. Developmental and stress regulation on expression of a novel miRNA, Fan-miR73, and its target ABI5 in strawberry. Scientific Reports. 2016 Jun;6:28385. doi:10.1038/srep2838.

9. Hartung W, Sauter A, Hose E. Abscisic acid in the xylem: where does it come from, where does it go to?. J. Exp. Bot. 2002 Jan;53(366):27-32.

10. Jiang $F$, Hartung $W$. Long-distance signalling of abscisic acid $(A B A)$ : the factors regulating the intensity of the $A B A$ signal. J. Exp. Bot. 2007 Jun;59(1):37-43.

11. Seo M, Koshiba T. Transport of ABA from the site of biosynthesis to the site of action. J. Plant Res. 2011 Jul;124(4):501-7.

12. Burla B, Pfrunder S, Nagy R, Francisco RM, Lee Y, Martinoia E. Vacuolar transport of abscisic acid glucosyl ester is mediated by ATP-binding cassette and proton-antiport mechanisms in Arabidopsis. Plant Physiol. 2013 Nov;163(3):1446-58.

13. Saito S, Hirai N, Matsumoto C, et al. Arabidopsis CYP707As encode (+)-abscisic acid 8'-hydroxylase, a key enzyme in the oxidative catabolism of abscisic acid. Plant Physiol. 2004 Apr;134(4):1439-49.

14. Zhu G, Liu W, Ye N, Liu R, Zhang J. Involvement of the abscisic acid catabolic gene CYP707A2 in the glucoseinduced delay in seed germination and post-germination growth of Arabidopsis. Physiol. Plant. 2011 Dec;143(4):375-84.

15. Ren, J, Sun L, Wu J, et al. Cloning and expression analysis of cDNAs for ABA 8'-hydroxylase during sweet cherry fruit maturation and under stress conditions. J. Plant Physiol. 2010 Nov;167(17):1486-93.

16. Kushiro T, Okamoto M, Nakabayashi K, et al. The Arabidopsis cytochrome P450 CYP707A encodes ABA 8'hydroxylases: key enzymes in ABA catabolism. The EMBO J. 2004 Apr;23(7):1647-56.

17. Ji K, Chen $P$, Sun $L$, et al. Non-climacteric ripening in strawberry fruit is linked to ABA, FaNCED2 and FaCYP707A1. Funct Plant Biol. 2012 May;39(4):351-7.

18. Galli V, Messias RS, Perin EC, Borowski JM, Bamberg AL, Rombaldi CV. Mild salt stress improves strawberry fruit quality. LWT-Food Sci. Technol. 2016 Nov;73:693-9.

19. Jia $\mathrm{H}$, Chai $\mathrm{Y}$, Li C, et al. Abscisic acid plays an important role in the regulation of strawberry fruit ripening. Plant Physiol. 2011 Set;157(1)188-99.

20. Xu Y, Charles MT, Luo Z, et al. Ultraviolet-C priming of strawberry leaves against subsequent Mycosphaerella fragariae infection involves the action of reactive oxygen species, plant hormones, and terpenes. Plant Cell Environ. 2019 Mar;42(3):815-31. 
21. Kondo $S$, Sugaya $S$, Sugawa $S$, et al. Dehydration tolerance in apple seedlings is affected by an inhibitor of ABA 8'-hydroxylase CYP707A. J. Plant Physiol. 2012 Fev;169(3):234-41.

22. Jia $\mathrm{H}$, Xie Z, Wang $\mathrm{C}$, et al. Abscisic acid, sucrose, and auxin coordinately regulate berry ripening process of the Fujiminori grape. Funct. Integr. Genomics. 2017 Jul;17(4):441-57.

2021 by the authors. Submitted for possible open access publication under the terms and conditions of the Creative Commons Attribution (CC BY NC) license (https://creativecommons.org/licenses/by-nc/4.0/). 